\title{
Personalized medicine in cancer: where are we today?
}

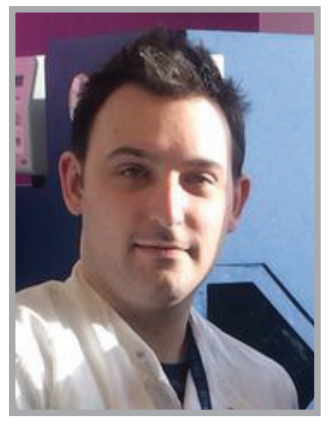

"As the field of translational medicine continues to expand and new targeted therapies and their associated tests become available, these will have to be integrated into the clinical care pathway to give clinicians the tools they need to make the most effective treatment decisions for each

Arran K Turnbull ${ }^{*, 1}$

What is personalized medicine? In an ideal world, our vision for personalized medicine involves measurement of some key tumor characteristics which inform the optimum therapy regimen tailored for each given patient to successfully treat their cancer and prevent relapse. In reality, things are not so simple. Cancer is enormously complex and heterogeneous and the current gaps in our knowledge preclude this vision from becoming a reality in the very near future. On the upside, groundbreaking advancements in technology and approaches in recent years have enabled tremendous strides in this direction, but we have a long way to go if this vision is to be fully realized.

\section{Where it all started}

In 1942, at Yale School of Medicine, two pharmacologists, Louis Goodman and Alfred Gilman, injected the first patient, a Polish immigrant metal worker known only by the initials JD who had advanced lymphoma and a large mandibular tumor, with a classified experimental substance designated as 'substance $\mathrm{X}$ ' in his medical records. Over the following weeks, they observed substantial tumor regression. Substance $\mathrm{X}$ was actually nitrogen mustard, a derivative of mustard gas discovered through chemical warfare research [1]. It worked by binding to the DNA of dividing cells resulting in cell death. This experiment was the first time a drug had been used to treat cancer and represented the beginnings of what we now call chemotherapy.

\section{The beginnings of personalized medicine}

The development and refinement of chemotherapy since then has revolutionized the treatment of cancer. Despite this, a large number of patients fail to respond to primary treatment or recur some time later, with the development of resistant malignancies. Thus the need for a personalized medicine approach was realized by the scientific community, in a move away from the 'one-treatment-fits-all' mindset $[2,3]$. To this day, breast cancer remains the most common cancer throughout the world, the most studied and best understood and will be the focus of this article exploring the concept of personalized medicine.

One of the most important discoveries in the field of cancer research was the estrogen receptor (ER) in breast cancer. While the dependence of breast cancers on the

'Edinburgh Breast Cancer Now Research Team, Institute of Genetics \& Molecular Medicine, University of Edinburgh \& Western General Hospital, Edinburgh, EH4 2XU, UK; Tel.: +44 0131537 1763; A.Turnbull@ed.ac.uk

\section{KEYWORDS}

- breast cancer • cancer

- personalized medicine 


\section{"High-throughput technologies such as gene-expression microarrays gave researchers the opportunity to explore the underlying genetics of cancer with a high degree of detail and accuracy."}

hormone estrogen has been well established for at least 100 years, the ER itself was only identified in 1958 by Elwood Jensen [4]. 20 years later, the first commercial endocrine therapy, tamoxifen, was available. It blocked the action of this receptor, revolutionizing the treatment of hormonedependent breast cancers with significantly less toxicity than its experimental predecessors. Prior to endocrine therapy, cessation of estrogen as a breast cancer treatment was achieved by ovarian oblation, as demonstrated by George Beatson in 1896. However, not all women gained noticeable benefit [5]. Discovery of the ER eventually led to the first molecular test for breast cancer [6]. For the first time, benefit from therapy could be predicted to some degree of accuracy; those women with detectable ER in diagnosis biopsies would gain benefit from endocrine therapy and those without would not. Indeed, to this day ER remains the most widely used marker of endocrine therapy benefit in breast cancer. Despite this, while it has a high negative predictive value, with virtually all ER-negative patients gaining no benefit from endocrine therapy, around $30-50 \%$ of those patients who are ER-positive will develop primary resistance or recur while on adjuvant therapy to prevent relapse [7].

\section{The dawn of the genomic age}

The latter part of the 20th century heralded the dawn of the genomic age. High-throughput technologies such as gene-expression microarrays gave researchers the opportunity to explore the underlying genetics of cancer with a high degree of detail and accuracy. In an effort to move toward a personalized approach, much work has been done to identify groups of patients with similar molecular characteristics which have significant associations with survival or drug response. In breast cancer, the pioneering work was done in 2001 by Sørlie, who, using gene-expression microarray technology, identified five distinct molecular subgroups of breast cancer with significantly different traits and survival associations [8]. The ER-positive population was subdivided into luminal A and B, the latter having significantly worse prognosis, and the ER-negative population was divided into basal, normal-like and HER2. The latter group expressed a cell surface receptor protein known as HER2, originally discovered in the 1980 s, which became the target of the drug Herceptin ${ }^{\circledR}[9,10]$. Now testing for HER2 is included alongside ER (and another hormone receptor, progesterone receptor $[\mathrm{PR}])$ as part of the routine diagnosis of breast cancer, and is the basis for prescription of Herceptin.

Since the initial work of Sørlie, the breast cancer molecular subtypes have been refined and redefined several times [11-14]. The increased accessibility of gene-expression microarrays and related high-throughput technologies, availability and use of public data repositories and the development of new bioinformatics approaches have all fueled the ever-expanding plethora of high-throughput genomic experiments. The majority of these have been concerned with profiling patients into groups with similar molecular characteristics associated with clinical end points such as prognosis or drug response. While this wealth of knowledge and data has revealed much about the underlying biology of cancer development, evolution and drug resistance, to date very few of these studies have been translated to the clinical setting to directly impact the management of breast cancer patients above and beyond the three markers (ER, PR and HER2) which are routinely used.

Only four prognostic/diagnostic tests for breast cancer currently have approval from the NICE for use in the UK, two of which, Oncotype $\mathrm{DX}^{\circledR}$ [15] and MammaPrint ${ }^{\circledR}$ [16], stemmed from large-scale genomic experiments. A third, IHC4 [17], uses immunohistochemistry (IHC) scoring of the three classic markers (ER, PR and HER2) plus Ki-67 and clinicopathological variables to estimate risk while the fourth, Mammostrat ${ }^{\circledR}$ [18], uses IHC measurement of five unique markers (SLC7A5, HTF9C, P53, NDRG1 and CEACAM5). All four tests seek to identify subsets of patients with such a good prognosis, where the absolute benefit is small compared with the associated toxicity, that they could forgo adjuvant systemic chemotherapy. However, there are a number of limitations associated with these tests. First, Oncotype DX, MammaPrint and Mammostrat are costly, at around US $\$ 2000-4000$ per patient and they require processing of tumor biopsy material in a central laboratory in the USA, which requires shipping of samples and can delay results by up to 10 days. All four tests have only been shown to have value in the ER-positive population of patients with no (or very few) positive lymph nodes and all four report results as a risk score subdivided into low-, medium- and high-risk groups, where the optimum clinical management of medium-risk patients remains unclear. 
Personalized medicine in cancer: where are we today? EDITORIAL

\section{Selecting patients for therapy}

The emergence of next-generation sequencing (NGS), with vastly improved throughput, speed, scalability and resolution, has ignited a revolution in genomic science allowing researchers to delve deeper into the genome, transcriptome and epigenome. While tests such as Oncotype DX and MammaPrint offer clinicians an important opportunity to make more effective use of systemic chemotherapy, the complexity and heterogeneity of cancer, now more apparent than ever before, has both highlighted the need and fueled the search for more targeted anticancer drugs.

Following on from Herceptin in 1998, we have seen a dramatic increase in the development of companion diagnostic tests in parallel with development of new targeted anticancer drugs. These tests detect particular characteristics of a cancer, such as specific proteins, transcripts or mutations, thereby selecting patients who are likely to gain benefit or not from the associated therapy. Targeted drug development of this type has not only increased the clinical success of new medications, by selecting specific subpopulations of patients where a given drug will be most effective, but has also afforded clinicians the first real opportunity to provide personalized care. As of July 2015, the US FDA has approved 18 companion diagnostic tests for use with specific anticancer drugs and this number will grow over the coming years. Indeed, multiple companion diagnostic tests can be approved for a single drug, as is now the case for Herceptin. This continued development and improvement of such tests is essential as the scientific knowledge evolves allowing for increased sensitivity and specificity. When we consider that ER, the most commonly used diagnostic test for endocrine therapy in breast cancer, has a sensitivity of only around $50-70 \%$, we can clearly appreciate the need for a more sophisticated test which can reliably and accurately predict response to therapy. Two tests which seek to do this, EndoPredict [19] and EndoResponse4 [20], not yet approved for clinical use, are currently in development.

\section{Making the most of targeted therapy}

Another important lesson from the laboratory has come from the study of cancer evolution, which has been aided significantly by deep sequencing technologies such as NGS. Recent studies have reported high numbers of heterogeneous gain and loss mutations which can occur between primary and distant sites and within primary tumors which develop drug resistance, drastically altering the biology of the cancer [21,22]. Based on these findings it seems clear that for optimal personalized care there is a need for clinicians, where possible, to take repeat biopsies of resistant tumors or biopsies of distant metastatic sites when considering targeted therapies. In many cases the cancer biology will have substantially changed and treatment decisions made on baseline diagnostic biopsies may no longer be effective. Indeed, in terms of predicting drug response, one recent study demonstrated the added value of an early on-treatment biopsy over baseline alone in predicting response to endocrine therapy, as the expression changes of some genes on therapy were more informative than their initial levels [20]. That said, while the anatomical location of breast tumors allows for routine biopsies to be easily performed under local anesthetic, this is not possible for some other cancers and distant metastatic sites. In time, improvements in blood testing including the isolation and analysis of circulating tumor cells may allow such tests to be performed noninvasively [23].

\section{The future}

Recent years have seen researchers and clinicians alike take great strides toward personalized cancer care. The advent and use of new high-throughput technologies has resulted in a data explosion in the field of cancer research. The challenge over the coming years will be to bridge the gap between the bench and bedside. Our goal should be to translate the findings of these large-scale studies back to the clinic to directly improve patient care through identification of new drug targets and continually developing and refining prognostic and diagnostic tests to determine which patients will benefit from which therapies. As the field of translational medicine continues to expand and new targeted therapies and their associated tests become available, these will have to be integrated into the clinical care pathway to give clinicians the tools they need to make the most effective treatment decisions for each patient.

\section{Financial \& competing interests disclosure}

The author has no relevant affliations or financial involvement with any organization or entity with a financial
"The advent and use of new high-throughput technologies has resulted in a data explosion in the field of cancer research.” 
interest in or financial conflict with the subject matter or materials discussed in the manuscript. This includes employment, consultancies, honoraria, stock ownership or options, expert testimony, grants or patents received or pending, or royalties.

No writing assistance was utilized in the production of this manuscript.

\section{Open access}

This article is distributed under the terms of the Creative Commons Attribution License 4.0 which permits any use, distribution, and reproduction in any medium, provided the original author(s) and the source are credited. To view a copy of the license, visit http://creativecommons.org/ licenses/by/4.0/

\section{References}

1 Gilman A. The initial clinical trial of nitrogen mustard. Am. J. Surg. 105, 574-578 (1963).

2 Holohan C, Van Schaeybroeck S, Longley DB, Johnston PG. Cancer drug resistance: an evolving paradigm. Nat. Rev. Cancer 13(10), 714-726 (2013).

3 Giaccone G, Pinedo HM. Drug resistance. Oncologist 1(1 \& 2), 82-87 (1996).

4 Jensen EV, Jordan VC. The estrogen receptor: a model for molecular medicine. Clin. Cancer Res. 9(6), 1980-1989 (2003).

5 Clarke MJ. Ovarian ablation in breast cancer, 1896 to 1998: milestones along hierarchy of evidence from case report to cochrane review. BMJ 317(7167), 1246-1248 (1998).

6 Jordan VC, Jacobson HI, Keenan EJ. Determination of estrogen receptor in breast cancer using monoclonal antibody technology: results of a multicenter study in the United States. Cancer Res. 46 (8 Suppl.), 4237s-4240s (1986).

7 Dixon JM. Endocrine resistance in breast cancer. New J. Sci. doi:10.1155/2014/390618 (2014) (Epub ahead of print).

8 Sorlie T, Perou CM, Tibshirani R et al. Gene expression patterns of breast carcinomas distinguish tumor subclasses with clinical implications. Proc. Natl Acad. Sci. USA 98(19), 10869-10874 (2001).

9 Slamon DJ, Clark GM, Wong SG, Levin WJ, Ullrich A, McGuire WL. Human breast cancer: correlation of relapse and survival with amplification of the $\mathrm{HER}-2 / \mathrm{Neu}$ oncogene. Science 235(4785), 177-182 (1987).

10 Delaney P. Her-2: the making of Herceptin, a revolutionary treatment for breast cancer. J. Natl Cancer Inst. 91(15), 1329-1330 (1999).

11 Sorlie T, Tibshirani R, Parker J et al. Repeated observation of breast tumor subtypes in independent gene expression data sets. Proc. Natl Acad. Sci. USA 100 (14), 8418-8423 (2003).

12 Perou CM, Sorlie T, Eisen MB et al. Molecular portraits of human breast tumours. Nature 406(6797), 747-752 (2000).

13 Prat A, Parker JS, Karginova O et al. Phenotypic and molecular characterization of the claudin-low intrinsic subtype of breast cancer. Breast Cancer Res. 12(5), R68 (2010).

14 Sorlie T. Molecular portraits of breast cancer: tumour subtypes as distinct disease entities. Eur. J. Cancer 40(18), 2667-2675 (2004).

15 Paik S. Development and clinical utility of a 21-gene recurrence score prognostic assay in patients with early breast cancer treated with tamoxifen. Oncologist 12(6), 631-635 (2007).

16 Van 'T Veer LJ, Dai H, Van De Vijver MJ et al. Gene expression profiling predicts clinical outcome of breast cancer. Nature 415(6871), 530-536 (2002).

17 Cuzick J, Dowsett M, Pineda S et al. Prognostic value of a combined estrogen receptor, progesterone receptor, $\mathrm{Ki}-67$, and human epidermal growth factor receptor 2 immunohistochemical score and comparison with the genomic health recurrence score in early breast cancer. J. Clin. Oncol. 29(32), 4273-4278 (2011).

18 Bartlett JM, Thomas J, Ross DT et al. Mammostrat as a tool to stratify breast cancer patients at risk of recurrence during endocrine therapy. Breast Cancer Res. 12(4), R47 (2010).

19 Filipits M, Rudas M, Jakesz R et al. A new molecular predictor of distant recurrence in ER-positive, HER2-negative breast cancer adds independent information to conventional clinical risk factors. Clin. Cancer Res. 17(18), 6012-6020 (2011).

20 Turnbull AK, Arthur LM, Renshaw L et al. Accurate prediction and validation of response to endocrine therapy in breast cancer. J. Clin. Oncol. 33(20), 2270-2278 (2015).

21 Stephens PJ, Tarpey PS, Davies $\mathrm{H}$ et al. The landscape of cancer genes and mutational processes in breast cancer. Nature 486(7403), 400-404 (2012).

22 Robinson DR, Wu YM, Vats P et al. Activating esr1 mutations in hormoneresistant metastatic breast cancer. Nat. Genet. 45(12), 1446-1451 (2013).

23 Punnoose EA, Lackner MR. Challenges and opportunities in the use of ctcs for companion diagnostic development. Recent Results Cancer Res. 195, 241-253 (2012). 